\title{
Evidence for strong electronic correlations in the spectra of $\mathrm{Sr}_{2} \mathrm{RuO}_{4}$
}

\author{
Z. V. Pchelkina, ${ }^{1, *}$ I. A. Nekrasov, ${ }^{2}$ Th. Pruschke, ${ }^{3}$ A. Sekiyama, ${ }^{4}$ S. Suga, ${ }^{4}$ V. I. Anisimov, ${ }^{1}$ and D. Vollhardt ${ }^{5}$ \\ ${ }^{1}$ Institute of Metal Physics, Russian Academy of Sciences-Ural Division, 620041 Yekaterinburg GSP-170, Russia \\ ${ }^{2}$ Institute of Electrophysics, Russian Academy of Sciences-Ural Division, 620016 Yekaterinburg, Russia \\ ${ }^{3}$ Institute for Theoretical Physics, University of Göttingen, Friedrich-Hund-Platz 1, D-37077 Göttingen, Germany \\ ${ }^{4}$ Graduate School of Engineering Science, Osaka University Toyonaka, Osaka 560-8531, Japan \\ ${ }^{5}$ Theoretical Physics III, Center for Electronic Correlations and Magnetism, University of Augsburg, D-86135 Augsburg, Germany
}

(Received 24 January 2006; revised manuscript received 5 October 2006; published 18 January 2007)

\begin{abstract}
We identify a satellite structure at $-3 \mathrm{eV}$ in the photoemission spectra of layered perovskite $\mathrm{Sr}_{2} \mathrm{RuO}_{4}$ as a lower Hubbard band. To this end we use state-of-the-art local-density approximation plus dynamical mean-field theory $(\mathrm{LDA}+\mathrm{DMFT})$ in the basis of Wannier functions to compute spectral functions and the quasiparticle dispersion of $\mathrm{Sr}_{2} \mathrm{RuO}_{4}$, and then compare the results with newly measured angle-integrated and angle-resolved spectra with 700-eV photon energy. Our theoretical spectra are in overall qualitative agreement with various spectroscopic experiments. We also calculate the $\mathbf{k}$ dependence of the quasiparticle bands and compare the results with high-energy bulk sensitive angle-resolved photoemission data.
\end{abstract}

DOI: 10.1103/PhysRevB.75.035122

PACS number(s): 71.27.+a, 79.60.-i

\section{INTRODUCTION}

Intensive research on $\mathrm{Sr}_{2} \mathrm{RuO}_{4}$ began after the discovery of superconductivity at temperatures below $1 \mathrm{~K}^{1}{ }^{1}$ Since it is widely believed that this system may help to clarify the mechanism behind high- $T_{c}$ superconductivity, considerable theoretical and experimental effort was put into the investigation of this material which has unconventional properties in spite of a relatively simple electronic structure.

It is generally recognized that in $3 d$ transition-metal compounds electron correlations play a crucial role. ${ }^{2}$ Since the $4 d$ states of the Ru ion are more extended than the $3 d$ states correlation effects in $\mathrm{Sr}_{2} \mathrm{RuO}_{4}$ should be less significant than, e.g., in high- $T_{c}$ cuprates. On the other hand, the effective quasiparticle mass obtained from de Haas-van Alphen $(\mathrm{dHvA}),{ }^{3}$ angle-resolved photoemission spectroscopy (ARPES), ${ }^{4}$ and infrared optical experiments ${ }^{5}$ is three to four times larger than the results obtained from standard band calculations. The temperature-independent contribution to the magnetic susceptibility and linear specific-heat coefficient ${ }^{1}$ are also significantly larger than that given by local-density approximation (LDA). ${ }^{6}$ These facts indicate that electron correlations do play an important role in $\mathrm{Sr}_{2} \mathrm{RuO}_{4}$.

One may identify two typical manifestations of strong correlations: (i) a considerable mass renormalization compared to results of single-electron theories, and (ii) satellite structures in the spectral function describing the lower Hubbard band (LHB). Since a mass enhancement can be induced by any many-body effect (for example, magnetic fluctuations, see Ref. 7) and not only by Coulomb correlation effects, the presence of a satellite in the spectra provides clear evidence for correlations, and is thus of particular importance for their identification.

During the last decade intensive studies of $\mathrm{Sr}_{2} \mathrm{RuO}_{4}$ using various spectroscopic techniques were performed. ${ }^{8-14}$ As to correlation effects the experimental results can be summarized as follows: (i) the density of states (DOS) at the Fermi level deviate by roughly a factor of 3 (Refs. 8 and 9) from band-structure calculations, ${ }^{6,15,16}$ and (ii) there is a peculiar satellite at $-3 \mathrm{eV}$ in the photoemission spectra (PES). ${ }^{11-14}$

Satellite structures in PES of transition-metal oxides which can be interpreted as the LHB are a direct manifestation of electronic correlation effects. Such satellites were first experimentally observed by Fujimori et al. ${ }^{17}$ for the $d^{1}$ perovskite-type $\mathrm{Ti}^{3+}$ and $\mathrm{V}^{4+}$ oxides. By applying the $a b$ initio LDA+DMFT (dynamical mean-field theory) approach $^{18-23}$ the corresponding structure in the many-body spectrum was later indeed identified as the LHB. . $^{18,20,24-26}$ While in $d^{1}$ compounds the $3 d$ band is usually well separated from the oxygen $2 p$ band (making the experimental observation and theoretical interpretation as the LHB relatively simple) the energy separation between the $\mathrm{Ru}-4 d$ and $\mathrm{O}-2 p$ states in $\mathrm{Sr}_{2} \mathrm{RuO}_{4}$ is much smaller (see below). In this case the LHB may overlap with the oxygen $2 p$ bands, making the interpretation of structures in the experimental spectra ambiguous. Differences in experimental conditions (photon energy, surface sensitivity, sample and surface quality, etc.) complicate the situation even more. Therefore interpretations of spectroscopic data for $\mathrm{Sr}_{2} \mathrm{RuO}_{4}$ are often controversial.

Early investigations of the electronic structure of $\mathrm{Sr}_{2} \mathrm{RuO}_{4}$ used LDA to reveal the similarities and differences with the electronic properties of cuprate superconductors. ${ }^{6,15}$ It was then proposed that the superconductivity of $\mathrm{Sr}_{2} \mathrm{RuO}_{4}$ may be unconventional, namely of triplet type $\mathrm{e}^{27,28}$ (see the comprehensive reviews ${ }^{29,30}$ ). A quantitative model for triplet superconductivity based on first-principles calculations for the electronic structure and magnetic susceptibility was suggested by Mazin and Singh. ${ }^{7}$ The electronic structure of $\mathrm{Sr}_{2} \mathrm{RuO}_{4}$ and $\mathrm{Sr}_{2} \mathrm{RhO}_{4}$ was compared in Ref. 16 and the possibility of a magnetic ground state of $\mathrm{Sr}_{2} \mathrm{RuO}_{4}$ was studied within the general gradient approximation $(\mathrm{GGA}){ }^{31}$

The Fermi surface of $\mathrm{Sr}_{2} \mathrm{RuO}_{4}$ was also investigated by LDA. According to these studies, the Fermi surface consists of three cylindrical sheets, ${ }^{6,15,16,32}$ in agreement with dHvA experiments. ${ }^{3}$ By contrast, ARPES experiments predicted a significantly different Fermi-surface topology. ${ }^{33-35}$ In principle, such a discrepancy may be due to strong electronic correlations which are not taken into account in LDA. How- 
ever, detailed photoemission studies ${ }^{4}$ and scanning-tunneling microscopy ${ }^{36}$ subsequently discovered a surface reconstruction which seemed to resolve the controversy. ${ }^{37,38}$

According to the results of band-structure calculations, a realistic description of $\mathrm{Sr}_{2} \mathrm{RuO}_{4}$ requires the examination of a general nondegenerate, multiorbital Hubbard model; see, e.g., Ref. 39 for early studies of such models within dynamical mean-field theory using quantum Monte Carlo as impurity solver $[\mathrm{DMFT}(\mathrm{QMC})]$. Consequently, Liebsch and Lichtenstein $^{40}$ used such a model in their attempt to clarify the discrepancy between dHvA (Ref. 3) and photoemission. $^{34,35}$ In their DMFT(QMC) calculations, they observed a charge transfer from the narrow $x z, y z$ bands to the wide $x y$ band leading to a shift of the van Hove singularity close to $E_{F}$, and derived quasiparticle bands by selfconsistent second-order perturbation theory for the selfenergy, finding a mass renormalization of $2.1-2.6,{ }^{40}$ in agreement with experiment. ${ }^{4,3}$ Anisimov et al. ${ }^{41}$ investigated the isoelectric series of alloys $\mathrm{Ca}_{2-x} \mathrm{Sr}_{x} \mathrm{RuO}_{4}$ by means of $\mathrm{LDA}+\mathrm{U}$ for $x=0$ and DMFT (non-crossing approximation) for $0.5<x<2.0$. In the latter doping range the scenario of an orbital selective Mott transition (OSMT) was proposed.

In this paper we investigate the satellite structure observed at $-3 \mathrm{eV}$ in the PES spectra of $\mathrm{Sr}_{2} \mathrm{RuO}_{4}$. A realistic $\mathrm{LDA}+\mathrm{DMFT}(\mathrm{QMC})$ calculation within a Wannier function (WF) formalism ${ }^{42}$ is employed. It allows us to take into account the influence of correlated orbitals $\left(4 d-t_{2 g}\right.$ orbitals of $\mathrm{Ru}$ in our case) on all other states. This is essential when $d$ and oxygen $p$ states overlap as is the case in $\mathrm{Sr}_{2} \mathrm{RuO}_{4}$. Our theoretical results show that electronic correlations indeed lead to the formation of a pronounced LHB which we identify with the satellite in the experimental spectra.

The paper is organized as follows. In Sec. II we present results for the band structure obtained by LDA (Sec. II A) and LDA+DMFT(QMC) in the WF basis (Sec. II B), respectively. Section III contains a comparison of our LDA + DMFT(QMC) results with XPS (x-ray photoemission spectroscopy), XAS (x-ray-absorption spectroscopy), and NEXAFS (near-edge x-ray fine structure) experiments, as well as with experimental PES (photoemission experiments) data (Sec. III A) and recent ARPES experiments (Secs. III B and III C). We conclude the paper with a summary, Sec. IV.

\section{ELECTRONIC STRUCTURE}

\section{A. LDA band structure}

$\mathrm{Sr}_{2} \mathrm{RuO}_{4}$ has the undistorted single-layered $\mathrm{K}_{2} \mathrm{NiF}_{4}$-type structure with the space group $14 / \mathrm{mmm}$ and lattice parameters $a=b=3.8603 \AA, c=12.729 \AA \AA^{43}$ The structure is formed by layers of $\mathrm{RuO}_{6}$ octahedra separated by $\mathrm{Sr}$ ions. The $\mathrm{RuO}_{6}$ octahedra are slightly elongated along the $c$ axis. Therefore the coordination of $\mathrm{Ru}$ ions locally has a tetragonal symmetry.

Our first-principle calculation of the electronic structure of $\mathrm{Sr}_{2} \mathrm{RuO}_{4}$ is based on density-functional theory (DFT) within the LDA approximation ${ }^{44,45}$ using the linearized muffin-tin orbitals (LMTO) method. ${ }^{46}$ The partial densities of states for $\mathrm{Sr}_{2} \mathrm{RuO}_{4}$ are shown in Fig. 1. They are in good agreement with results of previous calculations. $6,15,16,31,32$

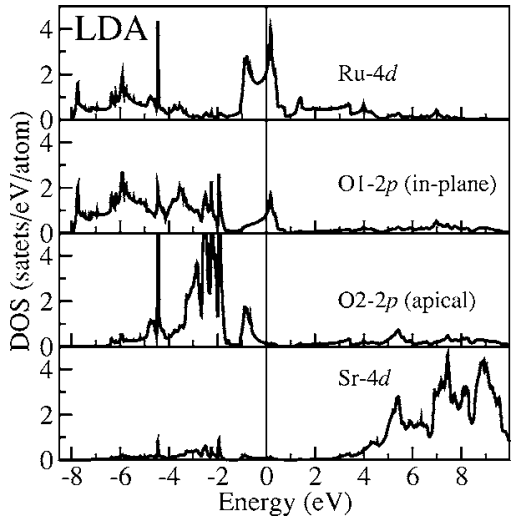

FIG. 1. Partial LDA DOS for $\mathrm{Sr}_{2} \mathrm{RuO}_{4}$. The Fermi level corresponds to zero.

The strontium $4 d$ states are almost empty and lie above $3 \mathrm{eV}$; the $\mathrm{O}-2 p$ derived bands are filled and extend from -8 to $-1 \mathrm{eV}$.

Physically most interesting are the partially filled ruthenium $4 d$ states. Due to the octahedral coordination of the oxygen ions, the Ru- $4 d$ states are split into $t_{2 g}$ and $e_{g}$ orbitals (see Fig. 2). Owing to the stronger hybridization of the two $e_{g}$ orbitals with oxygen $p$ states the corresponding bands lie above the three $t_{2 g}$ bands in the energy region from 0.5 to $5 \mathrm{eV}$. In $\mathrm{Sr}_{2} \mathrm{RuO}_{4}$ four $\mathrm{Ru}-4 d$ electrons occupy the three $t_{2 g}$ bands ( $d^{4}$ configuration). The layered crystal structure of $\mathrm{Sr}_{2} \mathrm{RuO}_{4}$ results in a two-dimensional DOS of the $x y$ orbital while the $x z, y z$ orbitals have nearly one-dimensional character (see Fig. 2). The $x y$ orbital hybridizes with $x y$ orbitals of the four Ru neighbors and thus has a bandwidth almost twice as large $\left(W_{x y}=2.8 \mathrm{eV}\right)$ as that of the $x z, y z$ orbitals $\left(W_{x z, y z}=1.5 \mathrm{eV}\right)$ which hybridize with corresponding orbitals of two Ru neighbors only.

The LDA bands in the energy window from -3 to $-1 \mathrm{eV}$ are shown in Fig. 3. In contrast to a typical $d^{1}$ system, ${ }^{26}$ there is no well-pronounced separation of the oxygen $2 p$ and ruthenium $4 d$ states in $\mathrm{Sr}_{2} \mathrm{RuO}_{4}$. More precisely, Fig. 3 shows that while the Ru- $4 d x y, y z$ orbitals are separated from the oxygen $2 p$ bands the Ru- $4 d x y$ orbital strongly overlaps with these oxygen bands.

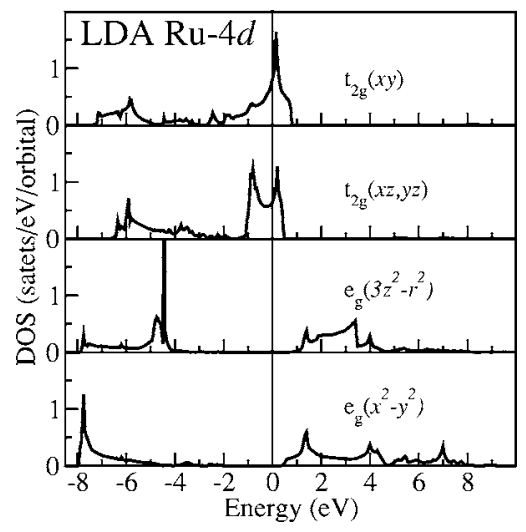

FIG. 2. Orbitally projected LDA Ru- $4 d$ DOS. The Fermi level corresponds to zero. 


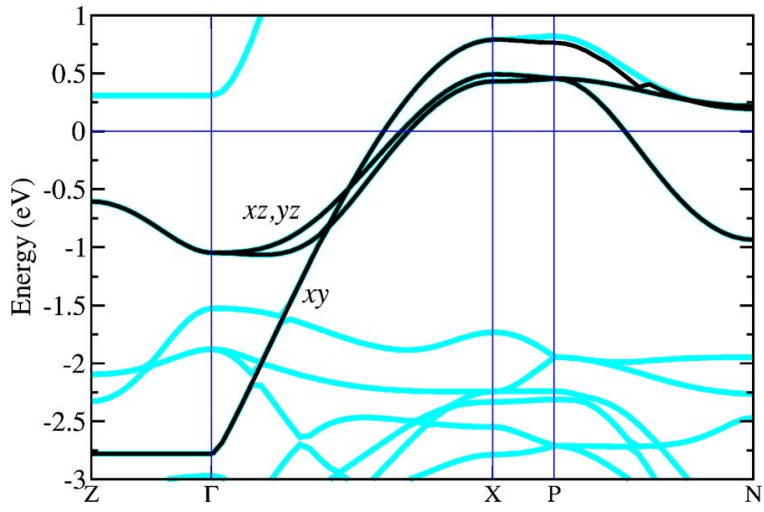

FIG. 3. (Color online) $\mathrm{Sr}_{2} \mathrm{RuO}_{4} \mathrm{LDA}$ band structure along high symmetry directions of the Brillouin zone. Light line: LDA bands; dark line: bands obtained by Wannier function projection on $t_{2 g}$ orbitals. The Fermi level corresponds to zero.

\section{B. LDA + DMFT(QMC) results: Effect of correlations}

$\mathrm{Sr}_{2} \mathrm{RuO}_{4}$ is a paramagnetic metal. ${ }^{1}$ It is well known that the paramagnetic state of a correlated metal is well described by the DMFT (for reviews, see Refs. 47-49). Within DMFT the electronic lattice problem is mapped onto a singleimpurity Anderson model with a self-consistency condition. ${ }^{50,51}$ This mapping, which becomes exact in the limit of large coordination number of the lattice, ${ }^{52}$ allows one to investigate the dynamics of correlated lattice electrons nonperturbatively at all interaction strengths. We use the LDA+DMFT $a b$ initio technique ${ }^{18-20,24}$ (for an introduction see, Ref. 23, for reviews see Refs. 21, 22, 53, and 54) to take into account correlation effects in $\mathrm{Sr}_{2} \mathrm{RuO}_{4}$. The effective impurity problem corresponding to the many-body Hamiltonian is solved by quantum Monte Carlo simulations. ${ }^{55}$ The LDA+DMFT approach was recently improved by employing a Wannier functions (WF) formalism, ${ }^{42}$ which allows one to project the Hamilton matrix from the full-orbital space to a selected set of relevant orbitals. The projection ensures that the information about all states in the system is kept. Other improvements of the LDA+DMFT method and formulation of more general spectral density-functional theory for electronic structure calculation of strongly correlated systems were introduced in the review of Kotliar et al. ${ }^{53}$ In the present work we use the WF formalism to construct an effective few-orbital Hamiltonian with $t_{2 g}$ symmetry and to take into account the influence of correlated $t_{2 g}$ orbitals on other states. A three-orbital Hamiltonian obtained by the WF projection with dispersions presented in Fig. 3 (black lines) was used as an $a b$ initio setup of the correlation problem. $A b$ initio values of the orbitally averaged Coulomb interaction parameter $\bar{U}=1.7 \mathrm{eV}$ and Hund exchange parameter $J$ $=0.7 \mathrm{eV}$ were obtained by constrained LDA calculations. ${ }^{56}$ We emphasize that not only were the on-site $e_{g}$ screening and the screening from $\mathrm{Ru}$ ions of the $\mathrm{RuO}_{2}$ plane taken into account in the calculation of the Coulomb interaction parameter, but also screening from neighboring $\mathrm{RuO}_{2}$ planes.

In the particular case of three $t_{2 g}$ orbitals $\bar{U}$ is equal to the interorbital Coulomb repulsion $U^{\prime} .^{21}$ Thus we obtain $U=U^{\prime}+2 J=3.1 \mathrm{eV}$ for the local intraorbital Coulomb repul-

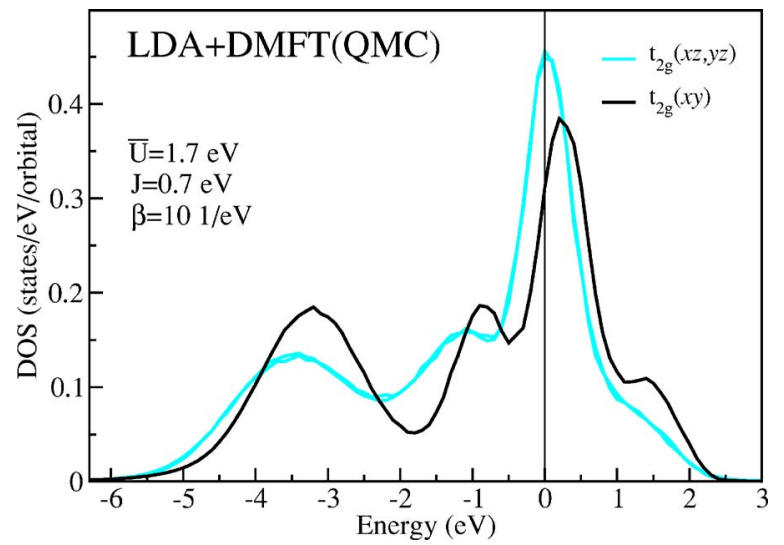

FIG. 4. (Color online) Ru-4 $\left(t_{2 g}\right)$ spectral functions obtained within LDA+DMFT(QMC) using a projected Hamiltonian. Dark curve: $x y$ orbital; light curve: $x z, y z$ orbitals; $\bar{U}=1.7 \mathrm{eV}, J$ $=0.7 \mathrm{eV}$. The Fermi level corresponds to zero.

sion. We note that our values of $U$ and $J$ differ substantially from those by Liebsch and Lichtenstein ${ }^{40}$ who in particular assumed a much smaller Hund exchange parameter. These authors estimated the Coulomb repulsion parameter from the XPS spectrum ${ }^{34}$ using the position of the resonance satellite. The value of about $1.5 \mathrm{eV}$ obtained thereby agrees well with our calculated value for $\bar{U}$. As is well known, $\bar{U}$ calculated from constrained LDA naturally contains uncertainties because the actual value sensitively depends on the screening channels included in its determination (for a more detailed discussion see, e.g., Ref. 20). On the other hand, Hund's exchange $J$ rather reflects the atomic multiplet structure, which usually is not very sensitive to changes of screening environment. 57

The three-orbital, projected Hamiltonian together with the $a b$ initio Coulomb interaction parameters were used as input for the QMC simulation of the effective quantum impurity problem arising in the DMFT. The simulations were performed for an inverse temperature $\beta=10 \mathrm{eV}^{-1}$ using 40 imaginary time slices $(\Delta \tau=0.25)$. Although the temperature chosen for the QMC calculations appears to be rather high, it is really sufficiently low, because it is much smaller than (i) the lowest atomic excitations (see the Appendix) and (ii) the characteristic low-energy scale $\sim 0.5 \mathrm{eV}$ obtained from the DMFT. The imaginary time QMC data were analytically continued by maximum entropy. ${ }^{58}$ The results are shown in Fig. 4. We find a pronounced lower Hubbard band (LHB) between -5 and $-1 \mathrm{eV}$, a quasiparticle peak (QP) around the Fermi level, and an upper Hubbard band (UHB) at about $1.5 \mathrm{eV}$. Real and imaginary parts of the corresponding selfenergy for real frequencies (for details see Appendix B in Ref. 42) for $t_{2 g}$ orbitals are shown in Fig. 5. Note that $\Sigma(\omega)$ displays Fermi-liquid behavior, i.e., the imaginary part of the self-energy has a parabolic maximum at the Fermi level. The rather large offset of $\operatorname{Im} \Sigma(0)$ is in accordance with $\operatorname{Im} \Sigma(0) \propto\left(\pi T / T_{K}\right)^{2}$ expected from Fermi liquid theory if one inserts $T=0.1 \mathrm{eV}$ and $T_{K} \approx 0.5 \mathrm{eV}$. The mass enhancement can be calculated from the derivative of $\operatorname{Re} \Sigma$ at the Fermi level and amounts to 2.62 for the $x y$ orbital and 2.28 for the $x z, y z$ orbitals, in agreement with results from ARPES, ${ }^{4}$ 


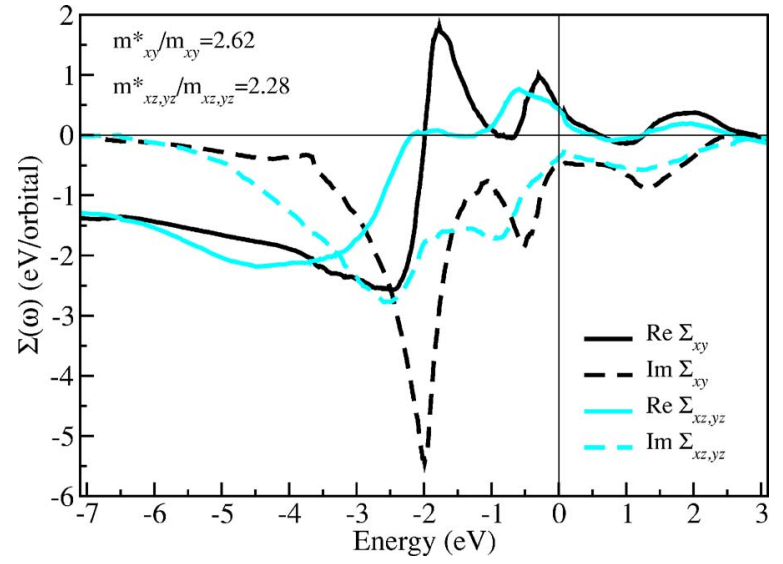

FIG. 5. (Color online) Self-energy on the real energy axis for $x y$ (black line) and $x z, y z$ (light line) orbitals of $\mathrm{Sr}_{2} \mathrm{RuO}_{4}$. Solid line: real part; dashed line: imaginary part. The Fermi level corresponds to zero.

$\mathrm{dHvA},{ }^{3}$ and infrared optical experiments. ${ }^{5}$ A detailed analysis of the structures seen in Fig. 4 is presented in the Appendix.

After having calculated the self-energy $\Sigma(\omega)$ for real frequencies one may perform the inverse transformation from the reduced Wannier basis back to the full LMTO basis. ${ }^{42}$ This step allows one to take into account the influence of the three correlated $t_{2 g}$ orbitals on all other states of ruthenium, oxygen, and strontium. The comparison of the noninteracting LDA partial density of states with the one obtained by the inverse transformation is shown in Fig. 6. Since the hybridization of $\mathrm{Ru}$ and $\mathrm{O}$ is quite strong the oxygen states are changed rather significantly by correlation effects on $\mathrm{Ru}$ ions. These changes are most pronounced in the energy region between -4 and $-1 \mathrm{eV}$. One can see that apical oxygen atoms are more affected by correlations than in-plane atoms. We believe that this is due to the one-dimensional character

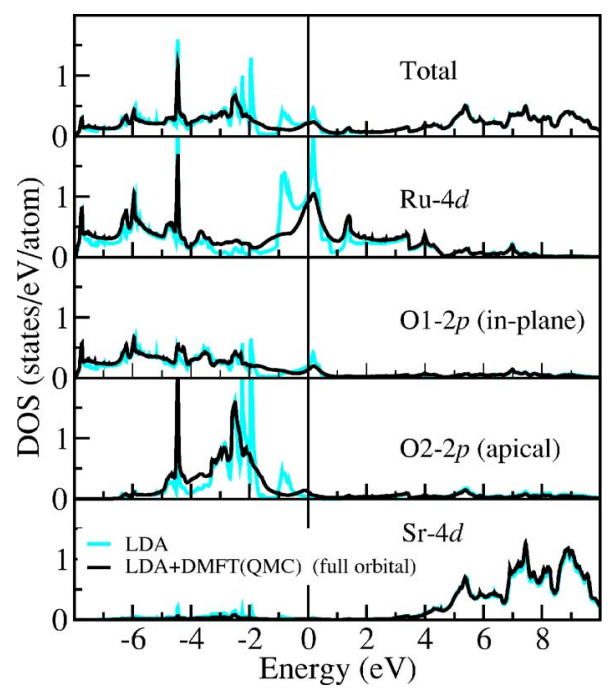

FIG. 6. (Color online) Comparison of the total and partial LDA DOS (light curve) and the DOS calculated using the full-orbital self-energy from LDA+DMFT(QMC) (black curve). The Fermi level corresponds to zero.

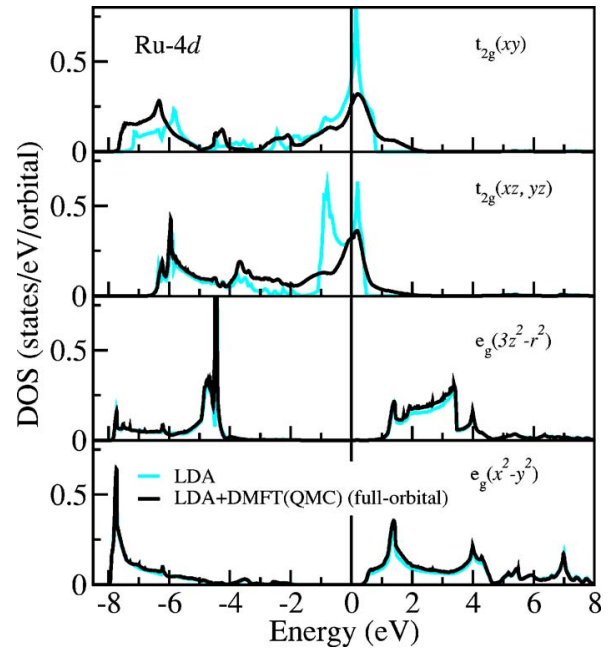

FIG. 7. (Color online) The same as in Fig. 6 but for Ru- $4 d$ states only.

of the $x z, y z$ states of the Ru- $4 d$ shell. Hence correlation effects are much stronger for these orbitals; consequently the DOS for the apical oxygen atoms is strongly modified. The $\mathrm{Sr}$ states are less affected. The significant modification of the oxygen states observed in our results (Fig. 6) means that in general oxygen orbitals should be explicitly included into the correlation problem. In our Wannier function approach we did this in an implicit way since oxygen orbitals gave a considerable contribution to the $t_{2 g}$ bands around the Fermi level (Fig. 1) and hence to the Wannier functions constructed from these bands. In the most general case it is not enough to include $\mathrm{O}-2 p$ states into the correlation problem. Basis orbitals set and charge density should be recalculated selfconsistently taking into account modifications due to the correlations from the LDA values. These points were also addressed in the review of Kotliar et al. ${ }^{53}$

A comparison between the partial LDA DOS of $\mathrm{Ru}-4 d$ and the DOS obtained using the full-orbital self-energy from our LDA+DMFT(QMC) calculations is shown in Fig. 7. The main effect of the correlations on the $\mathrm{Ru}-4 d$ states is seen to be a transfer of spectral weight from the energy region near the Fermi level to the lower and upper Hubbard bands range from -4 to $-1 \mathrm{eV}$, and from 1 to $2 \mathrm{eV}$, respectively.

\section{COMPARISON WITH EXPERIMENTAL DATA}

\section{A. XPS and PES experiments}

We will now compare the calculated LDA + DMFT(QMC) spectral functions for the $t_{2 g}$ electrons (light solid lines in Fig. 8-10 and those calculated using the fullorbital self-energy (thick black lines in the same figures) with several experimentally obtained spectra describing both valence and conduction bands. To compare with experiment we took into account the photoemission cross-section ratio for Ru- $4 d$ and $\mathrm{O}-2 p$ states as a function of photon energy. ${ }^{59}$ We found that, in general, an energy dependent broadening of the theoretical spectral functions gives better agreement 


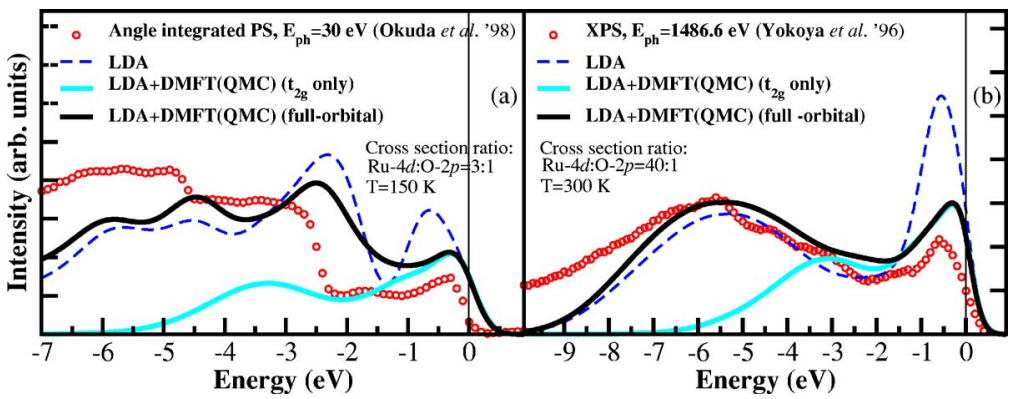

FIG. 8. (Color online) Theoretical spectral functions of $\mathrm{Sr}_{2} \mathrm{RuO}_{4}$ calculated by LDA+DMFT(QMC); light solid line: $t_{2 g}$ orbitals; black solid line: full-orbital self-energy. In (a) the results are compared to an angle-integrated valence-band photoemission spectrum (Ref. 10) obtained with a photon energy $E_{\mathrm{ph}}=30 \mathrm{eV}$, while in (b) we compare to an XPS spectrum obtained with a photon energy $E_{\mathrm{ph}}=1486.6 \mathrm{eV}$ (Ref. 11). The theoretical spectra are convoluted using a linear broadening $-0.04 E+0.25$ for (a), and $-0.14 E+0.25$ for (b) to account for the experimental resolution. Intensities are normalized on the area under curves. The Fermi level corresponds to zero.

with the experimental data (see Refs. 60 and 61). For this the theory curves were convoluted using a Gaussian with a full width at half maximum increasing as $C \cdot E+g$. Here $E$ is the binding energy, $g$ is the experimental resolution, and $C$ characterizes the increase of the broadening with energy upon moving away from Fermi level due to core-hole lifetime effects. The maximally allowed broadening was restricted to $1 \mathrm{eV}$. Specific values of $C$ and $g$ parameters used for comparison with experiment are indicated in the corresponding figures.

\section{Comparison with previous XPS experiments}

In Fig. 8 we compare an angle integrated valence-band photoemission spectrum of $\mathrm{Sr}_{2} \mathrm{RuO}_{4}{ }^{10}$ [Fig. 8(a)] and the XPS spectra from Ref. 11 [Fig. 8(b)] with the theoretical spectral functions. The contributions from $\mathrm{Ru}-4 d$ and $\mathrm{O}-2 p$ spectra were weighted according to the photoemission crosssection ratio ${ }^{59}$ 3:1 for Fig. 8(a), corresponding to a photon energy of $30 \mathrm{eV}$, and 40:1 for Fig. 8(b), corresponding to a photon energy of $1486.6 \mathrm{eV}$. The theoretical spectra were multiplied with the Fermi function at $T=150$ and $300 \mathrm{~K}$, respectively. In Fig. 8 a linear broadening was employed. UPS (ultraviolet photoemission) data of the valence band of $\mathrm{Sr}_{2} \mathrm{RuO}_{4},{ }^{8}$ obtained at photon energies of 60 and $110 \mathrm{eV}$, show similar features as the PES and XPS spectra in Fig. 8.

The experimental spectrum in Fig. 8(a) was obtained at a rather low photon energy of $30 \mathrm{eV}$. Therefore according to the cross-section ratio, the contribution of oxygen states is considerable. The positions and weight of peaks in the calculated curves and the experiment are in overall agreement. However, despite partially similar line shapes the theoretical curves have too much weight in the energy region of $-2 \mathrm{eV}$ in Fig. 8(a). This discrepancy can be attributed to an overestimation of the oxygen contribution in the theoretical curves, or to matrix element effects which may be significant at low photon energies. Nevertheless, the LDA+DMFT(QMC) spectra are seen to be in better agreement with experiment than the LDA results. In particular, in LDA+DMFT the LDA peak near $-0.5 \mathrm{eV}$ becomes a plateau [see Fig. 8(a)]-the redistribution of spectral weight being an effect of correlations. Comparing the theoretical $t_{2 g}$ and full-orbital spectra with experiment, one can see that the latter yield a better but still qualitative description in a wide energy range.

The XPS spectrum in Fig. 8(b) obtained at a very high photon energy is seen to be almost exclusively Ru- $4 d$ states. One can see that the full-orbital spectral function gives good agreement with the XPS data. Moreover, one observes a pronounced maximum at $-3 \mathrm{eV}$ which experimentalists previously interpreted as the LHB. ${ }^{11}$ This conjecture is now confirmed by our calculations (see the detailed discussion below).

\section{Comparison with new PES experiment}

Clean (001) surfaces of high-purity single-crystal samples were obtained by in situ cleavage at $20 \mathrm{~K}$ in ultrahigh vacuum. Angle-integrated and angle-resolved spectra were measured using the GAMMADATA-SCIENTA SES200 Analyzer at BL25SU of SPring-8 by use of circularly polarized light. Both measurements were performed at $700 \mathrm{eV}$ by detecting near normal emission electrons to obtain highest bulk sensitivity at this photon energy. The resolution for these measurements was set to $200 \mathrm{meV}$ (Fig. 9) and $120 \mathrm{meV}$ (Fig. 11), respectively. The energy scale was calibrated by the Fermi edge of Au. The surface cleanliness was checked by the absence of possible additional spectral weight on the higher binding energy side of the $\mathrm{O} 1 \mathrm{~s}$ peak, and by the absence of the $\mathrm{C} 1 s$ contribution.

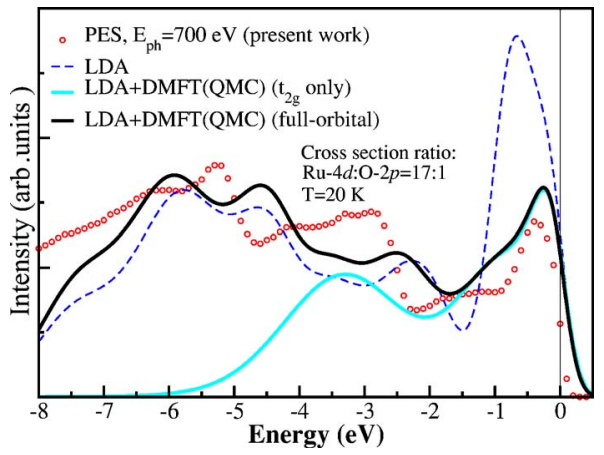

FIG. 9. (Color online) Similar plot as in Fig. 8 but now we compare with PES spectrum of $\mathrm{Sr}_{2} \mathrm{RuO}_{4}$. The theoretical spectra are convoluted using a linear broadening $-0.04 E+0.20$ to account for the experimental resolution. 


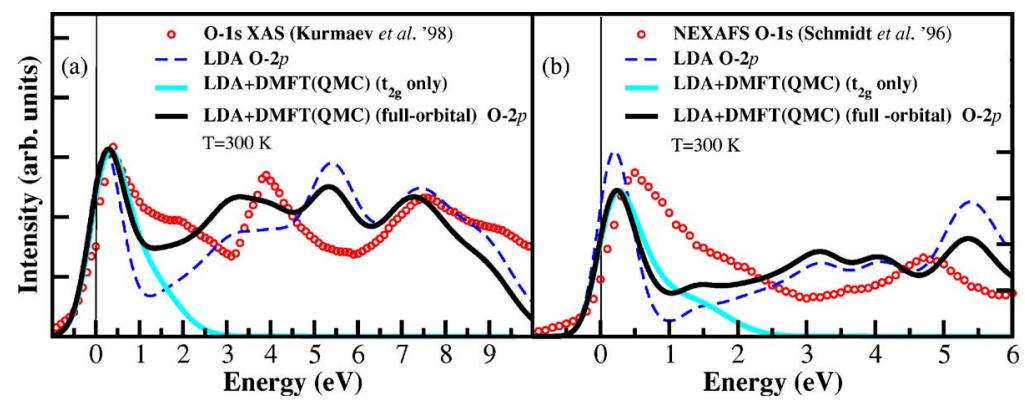

FIG. 10. (Color online) Theoretical spectra of $\mathrm{Sr}_{2} \mathrm{RuO}_{4}$ calculated by LDA+DMFT(QMC); light solid line: only $t_{2 g}$ electrons, black solid line: full-orbital self-energy using O- $2 p$ states. (a) Comparison with O- $1 s$ XAS spectrum (Ref. 9), (b) comparison with NEXAFS spectrum (Ref. 8). Theoretical spectra are convoluted using linear broadening $0.01 E+0.35$ for (a), and $0.02 E+0.2$ for (b) to account for the experimental resolution. Intensities are normalized on the area under curves. The Fermi level corresponds to zero.

In Fig. 9 we compare photoemission spectrum of $\mathrm{Sr}_{2} \mathrm{RuO}_{4}$ with spectral functions calculated by LDA+DMFT(QMC). A weighted sum of Ru- $4 d$ and $\mathrm{O}-2 p$ spectral functions according to the photoemission cross-section ratio 17:1 (Ref. 59) was used-corresponding to an experimental photon energy of $700 \mathrm{eV}$. Theoretical spectra were multiplied with the Fermi function at $20 \mathrm{~K}$ and were linearly broadened to account for the experimental resolution.

\section{Interpretation}

We now discuss and interpret the experimental and theoretical spectra, in particular in view of the presence of a LHB in the computed spectra. In Fig. 8(b) the structure in the experimental XPS spectrum at $-3 \mathrm{eV}$ was interpreted as the LHB. $^{11}$ In the full-orbital LDA+DMFT(QMC) spectral function (black solid line) a corresponding feature is indeed visible, but has less intensity and appears only as a shallow shoulder rather than a distinct bulge. There is no such a structure in the LDA spectral function (dashed line). Looking at the light solid line in Fig. 8(b) which represents the LDA+DMFT(QMC) DOS for the $t_{2 g}$ orbitals alone, we are able to identify this shoulder as a result of the LHB. Thus we have theoretically confirmed the interpretation of Yokoya et al. ${ }^{11}$ A very similar feature corresponding to the LHB in our LDA+DMFT(QMC) DOS was reported in Ref. 14.

The situation is similar in the PES spectrum. Because of lower photon energies, and due to the enhancement of the O- $2 p$ contribution in the PES spectrum seen in Fig. 9, one again cannot identify the $-3 \mathrm{eV}$ satellite directly. However, one can recognize a feature in the spectrum whose position coincides with the LHB in our LDA+DMFT(QMC) calculations. In the first theoretical DMFT work on the ruthenate, ${ }^{40}$ model-Coulomb parameters were chosen as $\bar{U}=0.8 \mathrm{eV}$ and $J=0.2 \mathrm{eV}$. As a consequence a less welldefined LHB with low spectral weight was obtained.

\section{B. XAS and NEXAFS experiments}

In Fig. 10(a) the O-1s X-ray-absorption spectrum (XAS) of $\mathrm{Sr}_{2} \mathrm{RuO}_{4}$ (Ref. 9) representing the conduction band is compared with the O- $2 p$ spectral function calculated via LDA+DMFT(QMC). Furthermore, Fig. 10(b) shows the near-edge $\mathrm{x}$-ray fine-structure spectrum (NEXAFS) of
$\mathrm{Sr}_{2} \mathrm{RuO}_{4},{ }^{8}$ together with the theoretical spectral functions. The theoretical spectra are multiplied with the Fermi function at $T=300 \mathrm{~K}$ and convoluted using linear broadening to account for the experimental resolution.

The agreement between theory and experiment in Fig. 10 is found to be qualitative. This may be due to empty states (conduction band) in the LMTO method. Namely, the conventional LMTO choice of the MTO linearization energy point lies inside the occupied part of the bands. As a result the unoccupied states in LDA calculation are not properly reproduced. Nevertheless, due to the spectral weight redistribution in the DMFT calculation, the agreement with experiment is improved (black solid line) in comparison with the bare LDA O- $2 p$ DOS (light dashed line).

\section{High-energy bulk ARPES experiment}

The comparison of experimental and theoretical spectra presented in the previous sections identifies the satellite structure in the photoemission spectra as a LHB. Let us consider the renormalization of quasiparticle properties, i.e., effective masses and band dispersions. This renormalization was addressed by Liebsch and Lichtenstein ${ }^{40}$ who derived

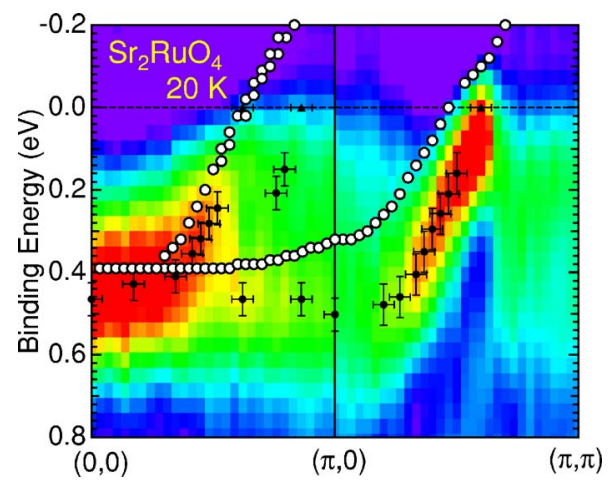

FIG. 11. (Color online) Comparison of the dispersion extracted from high-energy ARPES data (Ref. 67) obtained at $700 \mathrm{eV}$ under the off-resonance condition and LDA+DMFT(QMC). Experimental data contain the second derivative of the energy distribution curves, peak positions of the second derivative (black dots), and $k_{F}$ estimated from the momentum distribution curves at $E_{F}$ (triangles). The theoretical dispersion is indicated by open circles. 
quasiparticle bands from self-consistent second-order selfenergy. The Coulomb repulsion and Hund's rule exchange parameters chosen by these authors were rather small $(U=1.2-1.5 \mathrm{eV}, J=0.2-0.4 \mathrm{eV})$, resulting in a LHB with low spectral weight; nevertheless, the effective masses $\left(m^{*} \approx 2.1-2.6\right)$ were found to be in good agreement with experiments. In our investigation we determined the local Coulomb repulsion by the ab initio constrained LDA yielding $\bar{U}=1.7 \mathrm{eV}$ and $J=0.7 \mathrm{eV}$, i.e., a value of $U=3.1 \mathrm{eV}$ which is twice as large as that used in Ref. 40. This value of $U$ now produces a pronounced LHB and, at the same time, gives almost the same values of $\mathrm{m}^{*} / \mathrm{m}$ as those reported in Ref. 40: $\mathrm{m}^{*} / \mathrm{m}=2.62$ for the $x y$ orbital and 2.28 for the $x z, y z$ orbitals, respectively. All these values are in good agreement with experimental estimations..$^{3-5}$

We will now proceed to calculate $\boldsymbol{k}$-resolved spectra and quasiparticle band dispersions following the strategy proposed in Refs. 40, 62, and 63 and employed by us to calculate the ARPES spectra of $\mathrm{SrVO}_{3}{ }^{62}$ ARPES computations have previously been performed also for the twodimensional (2D) Hubbard model by Maier et al. ${ }^{64}$ in the framework of the dynamical cluster approximation (DCA), ${ }^{65}$ and by Sadovskii et al. ${ }^{66}$ within the so-called DMFT $+\Sigma_{k}$ approach.

To compare theoretical quasiparticle bands with the dispersion extracted from high-energy ARPES data we first calculate the $\boldsymbol{k}$-resolved spectral function $A(\boldsymbol{k}, \omega)$ for $\mathrm{Sr}_{2} \mathrm{RuO}_{4}$ (for details, see Ref. 62) defined by

$$
A(\boldsymbol{k}, \omega)=-\frac{1}{\pi} \operatorname{Im} \operatorname{Tr} \boldsymbol{G}(\boldsymbol{k}, \omega) .
$$

This quantity is determined by the diagonal elements of the Green-function matrix in orbital space

$$
\boldsymbol{G}(\boldsymbol{k}, \omega)=\left[\omega-\boldsymbol{\Sigma}(\omega)-\boldsymbol{H}_{0}^{\mathrm{WF}}(\boldsymbol{k})\right]^{-1},
$$

where $\boldsymbol{H}^{\mathrm{WF}}$ is the few-orbital Hamiltonian with $t_{2 g}$ symmetry obtained by WF projection. The corresponding eigenvalues are pictured in Fig. 3 as black lines. Since QMC only provides the self-energy $\Sigma$ for Matsubara frequencies $i \omega_{n}$ and the local spectrum $A(\omega)$, the calculation of $A(k, \omega)$ requires a method to compute $\Sigma$ for real frequencies $\omega$. This is achieved by first employing Kramers-Kronig to obtain

$$
G(\omega+i \eta)=\int_{-\infty}^{\infty} d \omega^{\prime} \frac{A\left(\omega^{\prime}\right)}{\omega-\omega^{\prime}+i \eta} .
$$

The local Green function and the complex self-energy are related by the $\boldsymbol{k}$-integrated Dyson equation

$$
\boldsymbol{G}(\omega)=\int_{\mathrm{BZ}} d \boldsymbol{k}\left[\omega+\mu-\mathbf{\Sigma}(\omega)-\boldsymbol{h}_{0}^{\mathrm{eff}}(\boldsymbol{k})\right]^{-1} .
$$

Solving Eq. (4) for given $G(\omega)$ for the self-energy $\Sigma(\omega)$ leads to the results presented in Fig. 5, which are then inserted into Eq. (2) to obtain the spectral function $A(\boldsymbol{k}, \omega)$ from Eq. (1). The maxima of $A(\mathbf{k}, \omega)$ obtained from this procedure are shown in Fig. 11 as open circles. Compared to experimental results our theoretical quasiparticle bands are shifted by $-0.08 \mathrm{eV}$.
ARPES data directly provide the energy distribution curves, ${ }^{67,68}$ whose second derivative represents the dispersion, which is shown in Fig. 11 by a "rainbow scale," red being the highest intensity. Closed circles with error bars denote the peak positions of the second derivative. The Fermi momenta $k_{\mathrm{F}}$, estimated from the momentum distribution curves (MDCs), ${ }^{67}$ at $E_{\mathrm{F}}$ are shown by triangles. We see that in the experiment there are two bands crossing the Fermi level at different $\boldsymbol{k}$ points in the $(0,0)-(\pi, 0)$ direction. There are also two bands in the calculated dispersion curve but they are almost degenerate. Nevertheless, the position of the bottom of quasiparticle bands, the intersection with the Fermi level, and the shape of the experimental and theoretical dispersions agree qualitatively.

\section{CONCLUSION}

In this paper we investigated $4 d$ resonance satellites in the theoretical and experimental spectra of the strongly correlated $4 d$ system $\mathrm{Sr}_{2} \mathrm{RuO}_{4}$. The electronic correlations lead to a typical redistribution of spectral weight and thereby to the formation of well pronounced lower and upper Hubbard bands (LHB, UHB). We note that on the experimental level the identification of a maximum in the spectrum of $\mathrm{Sr}_{2} \mathrm{RuO}_{4}$ is complicated by the overlap of the $\mathrm{Ru}-4 d$ and $\mathrm{O}-2 p$ bands.

We first calculated the electronic structure of $\mathrm{Sr}_{2} \mathrm{RuO}_{4}$ within the conventional band theory using LDA. The correlations were then taken into account in the framework of the $\mathrm{LDA}+\mathrm{DMFT}(\mathrm{QMC})$ scheme using $a b$ initio values for the Coulomb and Hund exchange parameters. We found that the ratio of Coulomb interaction and bandwidth is indeed larger than unity in $\mathrm{Sr}_{2} \mathrm{RuO}_{4}$, despite the rather extended $4 d$ states of $\mathrm{Ru}$, leading to a distinctive redistribution of spectral weight and to the formation of a well-pronounced LHB. Comparing our theoretical spectra with XPS experiments we identified this LHB with the structure observed at $-3 \mathrm{eV}$. By contrast, the LDA DOS shows no distinctive feature at that energy.

To describe the experimental spectra in a wide energy range we employed a Wannier function formalism to transform the self-energy operator back to the full-orbital basis. The theoretical spectra obtained in that way give a qualitative description of the high photon energy photoemission data; in particular, they reproduce the shoulder in the spectrum caused by the LHB. The basic features of the low photon energy UPS and intermediate energy PES spectra are also reproduced by the LDA+DMFT(QMC).

The calculated mass renormalization of about 2.5 agrees well with results from the previous DMFT(QMC) investigation by Liebsch and Lichtenstein ${ }^{40}$ and ARPES, dHvA, and infrared optical experiments. The LDA+DMFT(QMC) derived quasiparticle bands are in agreement with the dispersion extracted from ARPES data. However, in contrast to $\mathrm{LDA}+\mathrm{DMFT}(\mathrm{QMC})$ results for $3 d$ compounds, we found some clear discrepancies between calculated and experimental photoemission spectra of $\mathrm{Sr}_{2} \mathrm{RuO}_{4}$. The question whether these discrepancies are due to the influence of matrix elements or line-shape effects on the experimental spectra, or whether they must be interpreted as a deficiency of the the- 
TABLE I. Ground-state as well as single-particle excitations and their energies.

\begin{tabular}{ccccccc}
\hline \hline & $|x z\rangle$ & $|y z\rangle$ & $|x y\rangle$ & Energy $E_{\alpha}$ & Exitation energy $\epsilon$ & Value, eV \\
\hline $\mathrm{GS}$ & $|\uparrow \downarrow\rangle$ & $|\uparrow\rangle$ & $|\uparrow\rangle$ & $4 \epsilon_{0}+\Delta \epsilon+6 U-13 J$ \\
& & & occupied states \\
& & $\epsilon=E_{\mathrm{GS}}-E_{\alpha}$ & & -3.2 \\
$c_{x y}^{\uparrow}$ & $|\uparrow \downarrow\rangle$ & $|\uparrow\rangle$ & $|0\rangle$ & $3 \epsilon_{0}+3 U-5 J$ & $\epsilon_{0}+\Delta \epsilon+3 U-8 J=\epsilon_{0}+3.8$ & -1.9 \\
$c_{x z}^{\uparrow}$ & $|\downarrow\rangle$ & $|\uparrow\rangle$ & $|\uparrow\rangle$ & $3 \epsilon_{0}+\Delta \epsilon+3 U-7 J$ & $\epsilon_{0}+3 U-6 J=\epsilon_{0}+5.1$ & -3.3 \\
$c_{y z}^{\uparrow}$ & $|\uparrow \downarrow\rangle$ & $|0\rangle$ & $|\uparrow\rangle$ & $3 \epsilon_{0}+\Delta \epsilon+3 U-5 J$ & $\epsilon_{0}+3 U-8 J=\epsilon_{0}+3.7$ & -0.5 \\
$c_{x z}^{\downarrow}$ & $|\uparrow\rangle$ & $|\uparrow\rangle$ & $|\uparrow\rangle$ & $3 \epsilon_{0}+\Delta \epsilon+3 U-9 J$ & $\epsilon_{0}+3 U-4 J=\epsilon_{0}+6.5$ & \\
& & & & empty states & & 0.5 \\
& & & & $\epsilon=E_{\alpha}-E_{\mathrm{GS}}$ & & 0.4 \\
$c_{x y}^{\dagger}$ & $|\uparrow \downarrow\rangle$ & $|\uparrow\rangle$ & $|\uparrow \downarrow\rangle$ & $5 \epsilon_{0}+2 \Delta \epsilon+10 U-20 J$ & $\epsilon_{0}+\Delta \epsilon+4 U-7 J=\epsilon_{0}+7.5$ & $\epsilon_{0}+4 U-7 J=\epsilon_{0}+7.4$ \\
$c_{y z}^{\dagger}$ & $|\uparrow \downarrow\rangle$ & $|\uparrow \downarrow\rangle$ & $|\uparrow\rangle$ & $5 \epsilon_{0}+\Delta \epsilon+10 U-20 J$ & \\
\hline \hline
\end{tabular}

oretical method presently employed, cannot be answered at the moment and need to be clarified by future investigations.

\section{ACKNOWLEDGMENTS}

We thank R. Claessen, E. Z. Kurmaev, and A. Lichtenstein for helpful discussions. This work was supported by Russian Basic Research Foundation Grants No. RFFI-04-0216096, No. RFFI-05-02-17244, No. RFFI-05-02-16301, No. RFFI-06-02-81017, No. RFFI-BNTS-06-02-90537, by the Deutsche Forschungsgemeinschaft through Sonderforschungsbereiche 484 and 602, by MEXT Japan through 15GS0213 of Creative Scientific Research, by JSPS Japan through G18 of the 21st Century COE program, and in part by programs of the Presidium of the Russian Academy of Sciences (RAS) "Quantum macrophysics" and the Division of Physical Sciences of the RAS "Strongly correlated electrons in semiconductors, metals, superconductors and magnetic materials." I.N. and Z.P. acknowledge support by the Russian Science Support Foundation, Dynasty Foundation, and International Center for Fundamental Physics in Moscow. Z.P. also acknowledges the support from the Research Council of the President of the Russian Federation (Grant No. NSH-4192.2006.2). I.N. appreciates the support from the Grant of the President of the Russian Federation for Young Scientists, Grant No. MK-2118.2005.02.

\section{APPENDIX: ANALYSIS OF LDA+DMFT(QMC) SPECTRUM}

An important first step in interpreting the structures in the DOS in Fig. 4 is to identify purely atomic excitations. These will of course be shifted and broadened by correlation effects, but should, nevertheless, still be prominent. These excitations can readily be obtained from the atomic level picture. We denote the bare level energy of the $x z, y z$ orbitals as $\epsilon_{x z, y z} \equiv \epsilon_{0}$. Note that we do not know this value a priori, since it is neither the center of gravity of the bare LDA-DOS nor a particular peak position in the correlated DOS. It is, in fact, the unknown shift due to the double-counting correction of the LDA.

Hund exchange and Coulomb parameters from constrained LDA are $J=0.7 \mathrm{eV}$ and $\bar{U}=1.7 \mathrm{eV}$. For three $t_{2 g}$ orbitals we have $U^{\prime}=\bar{U}=1.7 \mathrm{eV}$ and thus $U=U^{\prime}+2 J$ $=3.1 \mathrm{eV}$. Finally, the splitting of the centers of gravity of the $x y$ and $x z, y z$ DOS is $\Delta \epsilon=\epsilon_{x y}-\epsilon_{x z, y z}=0.1 \mathrm{eV}$.

With these information we can construct the basis spanning the ground state and then calculate the excited states and their relative energies. Concentrating on a particular basis vector of the ground-state manifold, we obtain the scheme in Table. I, where we show the states contributing to the possible single-particle excitations. The corresponding excitation energies with the unknown level shift $\epsilon_{0}$ are listed in the third column. Obviously, the transition $C_{x y}^{\uparrow}|\mathrm{GS}\rangle$ represents the excitation with the lowest energy of the $x y$ DOS, i.e., should be identified with the position of lowest peak at energy $E_{1}=-3.2 \mathrm{eV}$ in the LDA+DMFT(QMC) DOS in Fig. 4 , leading to $\epsilon_{0}=-7 \mathrm{eV}$ and the final numerical values in the last column of Table I.

The identification of the further structures is now straightforward. For the $x y$ orbital, we must attribute the peak around $E=0.5 \mathrm{eV}$ to the excited states $c_{x y}^{\dagger}|\mathrm{GS}\rangle$. Likewise, in the $x z, y z$ manifold the peak at $E \approx-3.5 \mathrm{eV}$ corresponds to $c_{y z}^{\uparrow}|\mathrm{GS}\rangle$, and the broad structure at $E \approx-1.3 \mathrm{eV}$ to a superposition of $c_{x z}^{\uparrow}|\mathrm{GS}\rangle$ and $c_{x z}^{\downarrow}|\mathrm{GS}\rangle$. The peak at $E \approx 0 \mathrm{eV}$ finally can be identified with $c_{y z}^{\dagger}|\mathrm{GS}\rangle$.

Note that the peak in the $x y$ DOS at $E \approx-0.8 \mathrm{eV}$ has no correspondence in the atomic scheme. It could, however, be due to a finite admixture of the state $|\uparrow\rangle|\uparrow\rangle|\uparrow \downarrow\rangle$ to the ground state of the interacting system. This state would then allow for an excitation involving $c_{x y}^{\downarrow}$ with an excitation energy $-0.6 \mathrm{eV}$. 
*Electronic address: pzv@ifmlrs.uran.ru

${ }^{1}$ Y. Maeno, H. Hashimoto, K. Yoshida, S. Nishizaki, T. Fujita, J. G. Bednorz, and F. Lichtenberg, Nature (London) 372, 532 (1994).

${ }^{2}$ M. Imada, A. Fujimori, and Y. Tokura, Rev. Mod. Phys. 70, 1039 (1998).

${ }^{3}$ A. P. Mackenzie, S. R. Julian, A. J. Diver, G. J. McMullan, M. P. Ray, G. G. Lonzarich, Y. Maeno, S. Nishizaki, and T. Fujita, Phys. Rev. Lett. 76, 3786 (1996); A. P. Mackenzie, S. R. Julian, G. G. Lonzarich, Y. Maeno, and T. Fujita, ibid. 78, 2271 (1997).

${ }^{4}$ A. V. Puchkov, Z.-X. Shen, T. Kimura, and Y. Tokura, Phys. Rev. B 58, R13322 (1998).

${ }^{5}$ T. Katsufuji, M. Kasai, and Y. Tokura, Phys. Rev. Lett. 76, 126 (1996).

${ }^{6}$ T. Oguchi, Phys. Rev. B 51, 1385 (1995).

${ }^{7}$ I. I. Mazin and D. Singh, Phys. Rev. Lett. 79, 733 (1997).

${ }^{8}$ M. Schmidt, T. R. Cummins, M. Bürk, D. H. Lu, N. Nücker, S. Schuppler, and F. Lichtenberg, Phys. Rev. B 53, R14761 (1996).

${ }^{9}$ E. Z. Kurmaev, S. Stadler, D. L. Ederer, Y. Harada, S. Shin, M. M. Grush, T. A. Callcott, R. C. C. Perera, D. A. Zatsepin, N. Ovechkina, M. Kasai, Y. Tokura, T. Takahashi, K. Chandrasekaran, R. Vijayaraghavan, and U. V. Varadaraju, Phys. Rev. B 57, 1558 (1998).

${ }^{10}$ T. Okuda, H. Daimon, M. Kotsugi, K. Nakatsuji, M. Fujikawa, S. Suga, Y. Tezuka, S. Shin, M. Kasai, and Y. Tokura, J. Electron Spectrosc. Relat. Phenom. 88-91, 473 (1998).

${ }^{11}$ T. Yokoya, A. Chainani, T. Takahashi, H. Katayama-Yoshida, M. Kasai, Y. Tokura, N. Shanthi, and D. D. Sarma, Phys. Rev. B 53, 8151 (1996).

${ }^{12}$ I. H. Inoue, Y. Aiura, Y. Nishihara, Y. Haruyama, S. Nishizaki, Y. Maeno, T. Fujita, J. G. Bednorz, and F. Lichtenberg, Physica B 223-224, 516 (1996); I. H. Inoue, Y. Aiura, Y. Nishihara, Y. Haruyama, S. Nishizaki, Y. Maeno, T. Fujita, F. Lichtenberg, and J. G. Bednorz, J. Electron Spectrosc. Relat. Phenom. 78, 175 (1996).

${ }^{13}$ I. H. Inoue, A. Kimura, A. Harasawa, A. Kakizaki, Y. Aiura, S. Ikeda, and Y. Maeno, J. Phys. Chem. Solids 59, 2205 (1998).

${ }^{14}$ T. T. Tran, T. Mizokawa, S. Nakatsuji, H. Fukazawa, and Y. Maeno, Phys. Rev. B 70, 153106 (2004).

${ }^{15}$ D. J. Singh, Phys. Rev. B 52, 1358 (1995).

${ }^{16}$ I. Hase and Y. Nishihara, J. Phys. Soc. Jpn. 65, 3957 (1996).

${ }^{17}$ A. Fujimori, I. Hase, H. Namatame, Y. Fujishima, Y. Tokura, H. Eisaki, S. Uchida, K. Takegahara, and F. M. F. de Groot, Phys. Rev. Lett. 69, 1796 (1992).

${ }^{18}$ V. I. Anisimov, A. I. Poteryaev, M. A. Korotin, A. O. Anokhin, and G. Kotliar, J. Phys.: Condens. Matter 9, 7359 (1997).

${ }^{19}$ A. I. Lichtenstein and M. I. Katsnelson, Phys. Rev. B 57, 6884 (1998).

${ }^{20}$ I. A. Nekrasov, K. Held, N. Blümer, A. I. Poteryaev, V. I. Anisimov, and D. Vollhardt, Eur. Phys. J. B 18, 55 (2000).

${ }^{21}$ K. Held, I. A. Nekrasov, G. Keller, V. Eyert, N. Blumer, A. K. McMahan, R. T. Scalettar, Th. Pruschke, V. I. Anisimov, and D. Vollhardt, Psi-k Newsletter 56, 65 (2003), http://psi_k.dl.ac.uk/ newsletters/News_56/Highlight_56.pdf.

${ }^{22}$ K. Held, I. A. Nekrasov, N. Blümer, V. I. Anisimov, and D. Vollhardt, Int. J. Mod. Phys. B 15, 2611 (2001); K. Held, I. A. Nekrasov, G. Keller, V. Eyert, N. Blümer, A. K. McMahan, R. T. Scalettar, T. Pruschke, V. I. Anisimov, and D. Vollhardt, in Quantum Simulations of Complex Many-Body Systems: From Theory to Algorithms, edited by J. Grotendorst, D. Marks, and
A. Muramatsu, NIC Series Vol. 10 (NIC Directors, Forschunszentrum Jülich, 2002), pp. 175-209; A. I. Lichtenstein, M. I. Katsnelson, G. Kotliar, in Electron Correlations and Materials Properties 2nd ed., edited by A. Gonis, Nicholis Kioussis, and Mikael Ciftan (Kluwer Academic/Plenum, New York, 2002), p. 428.

${ }^{23}$ G. Kotliar and D. Vollhardt, Phys. Today 57, 53 (2004).

${ }^{24}$ M. B. Zölfl, Th. Pruschke, J. Keller, A. I. Poteryaev, I. A. Nekrasov, and V. I. Anisimov, Phys. Rev. B 61, 12810 (2000).

${ }^{25}$ E. Pavarini, S. Biermann, A. Poteryaev, A. I. Lichtenstein, A. Georges, and O. K. Andersen, Phys. Rev. Lett. 92, 176403 (2004)

${ }^{26}$ A. Sekiyama, H. Fujiwara, S. Imada, S. Suga, H. Eisaki, S. I. Uchida, K. Takegahara, H. Harima, Y. Saitoh, I. A. Nekrasov, G. Keller, D. E. Kondakov, A. V. Kozhevnikov, Th. Pruschke, K. Held, D. Vollhardt, and V. I. Anisimov, Phys. Rev. Lett. 93, 156402 (2004).

${ }^{27}$ T. M. Rice and M. Sigrist, J. Phys.: Condens. Matter 7, L643 (1995).

${ }^{28}$ G. Baskaran, Physica B 224, 490 (1996).

${ }^{29}$ A. P. Mackenzie and Y. Maeno, Rev. Mod. Phys. 75, 657 (2003).

${ }^{30}$ C. Bergemann, A. P. Mackenzie, S. R. Julian, D. Forsythe, and E. Ohmichi, Adv. Phys. 52, 639 (2003).

${ }^{31}$ P. K. de Boer and R. A. de Groot, Phys. Rev. B 59, 9894 (1999).

${ }^{32}$ G. J. McMullan, M. P. Ray, and R. J. Needs, Physica B 223-224, 529 (1996).

${ }^{33}$ D. H. Lu, M. Schmidt, T. R. Cummins, S. Schuppler, F. Lichtenberg, and J. G. Bednorz, Phys. Rev. Lett. 76, 4845 (1996).

${ }^{34}$ T. Yokoya, A. Chainani, T. Takahashi, H. Katayama-Yoshida, M. Kasai, and Y. Tokura, Phys. Rev. Lett. 76, 3009 (1996).

${ }^{35}$ T. Yokoya, A. Chainani, T. Takahashi, H. Ding, J. C. Campuzano, H. Katayama-Yoshida, M. Kasai, and Y. Tokura, Phys. Rev. B 54, 13311 (1996).

${ }^{36}$ R. Matzdorf, Z. Fang, Ismail, Jiandi Zhang, T. Kimura, Y. Tokura, K. Terakura, and E. W. Plummer, Science 289, 746 (2000).

${ }^{37}$ A. Damascelli, D. H. Lu, K. M. Shen, N. P. Armitage, F. Ronning, D. L. Feng, C. Kim, Z.-X. Shen, T. Kimura, Y. Tokura, Z. Q. Mao, and Y. Maeno, Phys. Rev. Lett. 85, 5194 (2000).

${ }^{38}$ A. Damascelli, Phys. Scr. 109, 61 (2004).

${ }^{39}$ M. J. Rozenberg, Phys. Rev. B 55, R4855 (1997).

${ }^{40}$ A. Liebsch and A. Lichtenstein, Phys. Rev. Lett. 84, 1591 (2000).

${ }^{41}$ V. I. Anisimov, I. A. Nekrasov, D. E. Kondakov, T. M. Rice, and M. Sigrist, Eur. Phys. J. B 25, 191 (2002).

${ }^{42}$ V. I. Anisimov, D. E. Kondakov, A. V. Kozhevnikov, I. A. Nekrasov, Z. V. Pchelkina, J. W. Allen, S.-K. Mo, H.-D. Kim, P. Metcalf, S. Suga, A. Sekiyama, G. Keller, I. Leonov, X. Ren, and D. Vollhardt, Phys. Rev. B 71, 125119 (2005).

${ }^{43}$ M. Braden, A. H. Moudden, S. Nishizaki, Y. Maeno, and T. Fujita, Physica C 273, 248 (1997).

${ }^{44}$ W. Kohn and L. J. Sham, Phys. Rev. 140, A1133 (1965); L. J. Sham and W. Kohn, Phys. Rev. 145, 561 (1966).

${ }^{45}$ L. Hedin and B. I. Lundqvist, J. Phys. C 4, 2064 (1971); U. von Barth and L. Hedin, ibid. 5, 1629 (1972).

${ }^{46}$ O. K. Andersen, Phys. Rev. B 12, 3060 (1975); O. Gunnarsson, O. Jepsen, and O. K. Andersen, Phys. Rev. B 27, 7144 (1983).

${ }^{47}$ D. Vollhardt, in Correlated Electron Systems, edited by V. J. Emery (World Scientific, Singapore, 1993), p. 57.

${ }^{48}$ Th. Pruschke, M. Jarrell, and J. K. Freericks, Adv. Phys. 44, 187 (1995).

${ }^{49}$ A. Georges, G. Kotliar, W. Krauth, and M. J. Rozenberg, Rev. 
Mod. Phys. 68, 13 (1996).

${ }^{50}$ A. Georges and G. Kotliar, Phys. Rev. B 45, 6479 (1992).

${ }^{51}$ M. Jarrell, Phys. Rev. Lett. 69, 168 (1992).

${ }^{52}$ W. Metzner and D. Vollhardt, Phys. Rev. Lett. 62, 324 (1989).

${ }^{53}$ G. Kotliar, S. Y. Savrasov, K. Haule, V. S. Oudovenko, O. Parcollet, and C. A. Marianetti, Rev. Mod. Phys. 78, 865 (2006).

${ }^{54}$ K. Held, cond-mat/0511293 (unpublished).

${ }^{55}$ J. E. Hirsch and R. M. Fye, Phys. Rev. Lett. 56, 2521 (1986); M. Jarrell, ibid. 69, 168 (1992); M. J. Rozenberg, X. Y. Zhang, and G. Kotliar, ibid. 69, 1236 (1992); A. Georges and W. Krauth, ibid. 69, 1240 (1992); M. Jarrell, in Numerical Methods for Lattice Quantum Many-Body Problems, edited by D. Scalapino (Addison-Wesley, Reading, MA, 1997).

${ }^{56}$ O. Gunnarsson, O. K. Andersen, O. Jepsen, and J. Zaanen, Phys. Rev. B 39, 1708 (1989); V. I. Anisimov and O. Gunnarsson ibid. 43, 7570 (1991).

${ }^{57}$ O. Gunnarsson and R. O. Jones, J. Chem. Phys. 72, 5357 (1980).

${ }^{58}$ For a review on the maximum entropy method, see Mark Jarrell and J. E. Gubernatis, Phys. Rep. 269, 133 (1996).

${ }^{59}$ J. J. Yeh and I. Lindau, Atomic Data and Nuclear Data Tables (Academic, New York, 1985), Vol. 32(1).

${ }^{60}$ L. Z. Liu, J. W. Allen, O. Gunnarsson, N. E. Christensen, and O. K. Andersen, Phys. Rev. B 45, 8934 (1992).

${ }^{61}$ A. J. Arko, J. J. Joyce, L. Morales, J. Wills, J. Lashley, F. Wastin, and J. Rebizant, Phys. Rev. B 62, 1773 (2000).

${ }^{62}$ I. A. Nekrasov, K. Held, G. Keller, D. E. Kondakov, Th. Prus- chke, M. Kollar, O. K. Andersen, V. I. Anisimov, and D. Vollhardt, Phys. Rev. B 73, 155112 (2006).

${ }^{63}$ S. Biermann, A. Dallmeyer, C. Carbone, W. Eberhardt, C. Pampuch, O. Rader, M. I. Katsnelson, and A. I. Lichtenstein, Pis'ma Zh. Eksp. Teor. Fiz. 80, 714 (2004); [JETP Lett. 80, 612 (2004)].

${ }^{64}$ Th. A. Maier, Th. Pruschke, and M. Jarrell, Phys. Rev. B 66, 075102 (2002).

${ }^{65}$ C. Huscroft, M. Jarrell, Th. Maier, S. Moukouri, and A. N. Tahvildarzadeh, Phys. Rev. Lett. 86, 139 (2001); M. H. Hettler, M. Mukherjee, M. Jarrell, and H. R. Krishnamurthy, Phys. Rev. B 61, 12739 (2000); T. Maier, M. Jarrell, T. Pruschke, and J. Keller, Eur. Phys. J. B 13, 613 (2000); M. H. Hettler, A. N. Tahvildar-Zadeh, M. Jarrell, T. Pruschke, and H. R. Krishnamurthy, Phys. Rev. B 58, R7475 (1998); Th. Maier, M. Jarrell, Th. Pruschke, and M. H. Hettler, Rev. Mod. Phys. 77, 1027 (2005).

${ }^{66}$ M. V. Sadovskii, I. A. Nekrasov, E. Z. Kuchinskii, Th. Pruschke, and V. I. Anisimov, Pis'ma Zh. Eksp. Teor. Fiz. 82, 217 (2005); [JETP Lett. 82, 198 (2005)].

${ }^{67}$ A. Sekiyama, S. Kasai, M. Tsunekawa, Y. Ishida, M. Sing, A. Irizawa, A. Yamasaki, S. Imada, T. Muro, Y. Saitoh, Y. Onuki, T. Kimura, Y. Tokura, and S. Suga, Phys. Rev. B 70, 060506(R) (2004).

${ }^{68}$ A. Sekiyama and S. Suga, J. Electron Spectrosc. Relat. Phenom. 137-140, 681 (2004). 